schon seit längerer Zeit, ähnliche Bestrebungen, die durch den Weltkrieg zweifellos auch besonders gefördert werden dürften, durchzusetzen und besonders der Chemie das Verständnis weiter Kreise zu erschließen. Der Bromberger Oberlehrer $I_{\mathbf{L}}$ Trink w a 1 t e $r$ hat in einem sehr beachtenswerten Aufsatz über: ,F r a g e n des chemischen Unterrichts" in der Zeitschrift: „Aus der Natur" (1917, S. 380-387 und 456-463) nicht mit Unrecht darauf hingewiesen, da $B$ man auch die führenden Geister der Chemio nicht ganz von den Vorwurf freispreohen könne, daß sie sich zu wenig bemühten, weitere Kreise für die Bedeutung chemischer Forschung zu interessieren; und auch seine weitere Bomerkung, $\mathrm{daB}$ die Vertreter anderer Wissenschaften und der Kunst den Chemikern auf dem Gebiet einer gesunden Popularisierung der Wissenschaft weit über seien, entbehrt durchaus nicht einer gewissen Berechtigung. Andererseits darf man aber auch nicht verkennen, $\mathrm{da} B$ in neuerer Zeit das Interesse für die Chemie in größeren Kreisen etwas gewachsen ist. Allerdings gibt es auch heute noch manche Chemiker, die allen solohen Bestrebungen mit einem gewissen Mißtrauen gegenüberstehen, weil sie daraus eine Gefährdung des rein wissenschaftliohen Charakters der Chemie befürchten.

Was Prof. K la t $t$ über die Bedeutung der Naturwissenschaften und besonders der Chemie für die staatsbürgerliche Erziehung sagt, die nach seinen Wünschen schon auf der Schule einsetzen sollte, enthält ebenfalls eine ganze Reihe von Anregungen, denen auch derjenige seine Zustimmung nicht versagen wird, der eine allgemeine Einführung in die Volkswirtschaft weniger der Sohule als den Hoohschulen zur Aufgabe stellen möchte. Ganz richtig wird allerdings hervorgehoben, daß die großen Mächte unserer Volkswirtschaft, Landwirtschaft und Industrie, ihre Bedeutung, ihre Bedürfnisse und ihre gegenseitigen Abhängigkeitsverhältnisse nur derjenige verstehen kann, der über einige naturwissenschaftliche Kenntnisse verfügt. ,Eine Durohdringung der ohemisch-technischen Tatsachen mit volkswirtschaftlichen Gesichtspunkten, das ist in der Tat das Ideal, dem auch der Technologe wird nachstreben müssen, der im Unterricht auf der Hochsohule auch auf die Angehörigen anderer Berufe wird einwirken wollen." Bis jetzt ist ein Versuch, die Volkswirtschaft im naturkundlichen und chemischen Unterricht darzustellen, noch nicht in befriedigender Weise gemacht worden. Es ist aber sehr beachtenswert, daB jetzt auch aus den Kreisen der Lehrerschaft heraus der Wunsch geäußert wird, eine solche Anleitung zu erbalten.

Man wird in der Tat unter Berüoksiohtigung der leider feststehenden Tatsache, daB auch im deutschen Volk die Kenntnisse von den Errungensohaften der Technik und der Industrie nooh viel zu wenig verbreitet sind, immer wieder darauf aufmerksam machen müssen, daß es, wie Klatt sehr richtig sagt, $n$ i c b t di e ro be Kraft der Arbeitsfäuteallein ist, dieDeutsoblands Industrie geschaffen hat, sonderndaB es vor allem geistige Krätegewesensind, denen man den Aufsohwung von Handel und Industrie z u verdanke $n$ at. Nicht nur die jetzt so beliebte Chemie des Krieges gilt es kennen zu lernen, sondern den Versuch zu machen, dem Sohüler und Studenten mit Hilfe einiger passend ausgewählter Beispiele aus der Geschichte der ohemischen Industrie einen Einbliok in dieses gewaltige Gebiet nationaler Arbeit zu verschaffen. Wenn man dadurch zu dem Ergebnis gelangt, Deutschlands Stellung in der Weltwirtsohaft frei von chauvinistisohen Vorurteilen allmählich zu begreifen, so bedeutet das in der Tat auoh ein Stück nationaler Erziehung.

Herr Prof. K la t t, der mit seinen Schülern von Görlitz aus vielfach technische Betriebe besichtigt hat, hält es auch für notwendig, bei der vorherigen Erörterung dieser Besichtigungen das soziale Moment nicht zu vergessen. Warmherziges soziales Empfinden $\mathrm{zu}$ wecken, ist sicherlich ebenfalls eine vaterländisch wertvolle Arbeit, die in den obersten Klessen, aber wohl besser noch auf den Universitäten und technischen Hochschulen, wie auch auf den Handelshoohschulen, niemals vergessen werden sollte, da doch gerade die deutsche Sozialpolitik den anderen Völkern als ein vor dem Kriege auoh im feindlichen Auslande stets neidlos anerkanntes Vorbild gegolten hat, ein Urteil, das auch heute noch seine volle Bereohtigung besitzt.

Wir werden uns zweifellos auch nach dem Kriege mit den Fragen des chemisohen Unterriohts auf den Schulen und Hochsohulen eingehend befassen müssen. Dabei wird es vor allem auch erforderlich sein, darauf hinzuarbeiten, daB die jetzt leider noch verhaltnismäbig losen Beziehungenzwischen den a kademischen Lehrern und den Schulpada. gogen im engeren Sinnefestergeknüpftwerd e $\mathrm{n}$, denn nur duroh eine gemeinsame Arbeit wird es sohließlich gelingen können, die einer notwendigen Erweiterung des naturwissensohaftlichen und speziell des chemischen Unterrichts entgegenstehenden Schwierigkeiten im allgemeinen Interesse zu überwinden.

[A. 96.]

\section{Glycerinbestimmung nach dem Jodidverfahren mit kleinen Mengen Jodwasserstoffsäure (Halb- mikrojodidverfahren).}

\author{
Von R. NHUMare.
}

(Mitteilung aus dem chemischen Laboratorium des Apollowerkes Wien-Simmering.)

Die quantitative Bestimmung des Glycerins nach dem Verf. von $\mathrm{Z}$ e i s e $\mathrm{l}$ - F a $\mathrm{n} \mathrm{t}^{1}$ ) konnte sich trotz mancher Vorzüge gegenüber anderen Glycerinbestimmungsmethoden keinen allgemeinen Eingang in die chemische Praxis verschaffen. Es wurde ihr vielfach die verhältnismäßig lange Ausführungsdauer und insbesonders der hohe Preis der in Betracht kommenden Reagenzien zum Vorwurfe gemacht, und man fand sie daher als technische Untersuchungsmethode ungeeignet. In den gegenwärtigen Kriegszeiten kommt neben den noch höheren Kosten der Chemikalien auch die schwere Beschaffungsmöglichkeit der Jodwasserstoffsäure hinzu, was ein konstantes Arbeiten nach dem Jodidverfahren völlig unmöglich macht.

Die Bestrebungen gingen infolgedessen dahin, den Verbrauoh an Jodwasserstoffsäure wesentlich zu verringern, und es war der Gedanke naheliegend, durch Umgestaltung der Jodidmethode in ein Mikroverf. zum Ziele zu gelangen. Eine Mikroanalyse im wahrsten Sinne des Wortes wäre für technische $Z$ wecke selbstverständlich undenkbar, da unseren Betriebs- und Untersuchungslaboratorien wohl sohwerlich eine Mikrowage zur Verfügung steht, ja in den meisten Fällen die Unterbringung einer solchen infolge Mangel an geeigneten Räumlichkeiten ganz unmöglich wäre. Es blieb somit zur Lösung dieser Aufgabe nur der Weg, bei möglichster Herabsetzung der Jodwasserstoffmenge die Konzentration der zu untersuchenden glycerinhaltigen Substanz derart zu wählen, daB das Gewicht des gebildeten Jodsilbers auf einer gewöhnlichen, guten, analytischen Wage wie sie sich in jedem besser eingerichteten chemischen Laboratorium vorfindet - festgestellt werden kann, um daraus mit genügender Genauigkeit den Glyceringehalt zu ermitteln.

Fine Anzahl im hiesigen Laboratorium ausgeführter Versuche hat gezeigt, da $B$ das Jodidverfahren bei Benutzung beliebig geringerer Mengen der Probesubstanz und entsprechend herabgesetzter Quantitäten der Reagentien ebenso gut verwendbar ist wie bei genauer Finhaltung der von $\mathrm{Z}$ e is el-Fanto angegebenen Arbeitsweise. So wurden zum Beispiel statt der vorgeschriebenen $5 \mathrm{com}$ bloB 2 com verschiedenster Glycerinlösungen mit nur 6 (statt 15) ccm Jodwasserstoffsüure (D. 1,98) im S t r i t a r schen Jodidapparate ${ }^{2}$ ) dem Jodidverfahren unterworfen (wobei gleichzeitig auch die entsprechend geringere Menge alkoholische Silbernitratlösung - $18 \mathrm{ccm}$ - verwendet wurde). In allen diesen Fällen erhielt man vollständig zufriedenstellende Resultate; eine wesentliche Abkürzung der Arbeitszeit wurde dadurch nicht erzielt.

Weit ökonomischer und vorteilhafter hingegen ist es, zur Jodidbestimmung nur den 10. Teil der Probelösung und der Reagenzien zu verwenden und deren Durchführung in einem eigens für diesen Zweck in verkleinertem Maßstabe angefertigten $S t r i t$ a $r$ schen Jodidapparate vorzunehmen. Dadurch wird nicht nur eine große Ersparnis an Material, sondern auch ein wesentlicher Gewinn an Zeit erzielt, was eine bedeutende Verbilligung der Methode darstellt.

1. De r A p para t. Der Apparat zur Ausführung der im nachfolgenden geschilderten "Halbmikrojodidbestimmung " ist genau so beschaffen wie der von $S$ trit a r konstruierte Jodidapparat, nur wurde er für den vorliegenden Zweck in den halben linearen Dimen. sionen hergestellt, so daB der Rauminhalt des Siedekölbchens und der Voriagen etwa den 8. Teil beträgt (siehe Abb. 1) ${ }^{3}$ ).

In besonderen Fällen ist es vorteilhaft, zwischen den Teilen $b$ und $c$ ein Absorptionsgefä $B g$ in Gestalt eines $S$ t $r$ i t a r schen Kaliappa-

1) Z. f. landw. Versuchswesen in Österr. 5, 729 [1902]; Z. f. analyt. Chemie 42, 549, 579 [1903]; Angew. Chem. 16, 413 [1903].

2) Z. anal. Chem. 42, 579 [1903]

s) Der Apparat ist gesetzlich geschützt und wird von der Firma $P$ a u l Ha a ck, Wien, IX. Garelligasse 4. hergestellt. 
rates') einzuschalten, wodurch der Jodidapparat die in Abb. 2 veranschaulichto Form annimmt.

2. A usführung der Operation. Die Ausführung der Bestimmung geschieht in ganz analoger Weise wie bei der bisher üblichen Art der Jodidbestimmung. Der Aufsatz des Teiles $b$ wird mit einer Aufschlämmung von $0,03-0,05$ g gereinigtem, dunkelrotem Phosphor in etwa 0,5 ccm Wasser gefüllt, welche Menge für eine sehr große Anzahl von Bestimmungen ausreicht. In die erste Vorlage $d$ gelangen etwa 4,5-5 com, in die zweite $\left.\mathrm{e}^{5}\right) 0,5 \mathrm{ccm}$ alkoholische Silbernitratlösung. In das Siedekölbohen (a) bringt man neben

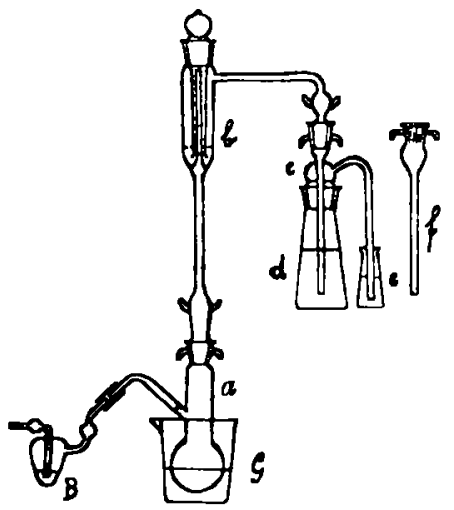

b . . Steigrohr mit Aufsatz samt Stopfen (Waschapparat).

Abb. 1. (1/3 der natürlichen Größe.)

1--2 kleinen Tonsplitterchen 0,5 cem der zu untersuchenden Lösung, die vorher auf einen Glyceringehalt von ungefähr 2,5-5\% gebracht werden muß, und hierauf $1,5 \mathrm{cem}$ Jodwasserstoffsäure vom spez. Gew. 1,9. Die Kohlensäure wird am zweckmäßigsten einer Stahlflasche, die mit einem Reduzierventil versehen ist, entnommen und vor Eintritt in den Apparat durch einen kleinen Blasenzähler $B$ geleitet (2-3 Blasen in der Sekunde).

Sohw efelhaltige Substanzen kann man auch o h n e die von $Z$ e isel-Fanto angegebene Vorreinigung dem Jodidverfahren unterwerfen, was eine bedeutende Vereinfachung in der Ausführung der Glycerinbestimmung darstellt. Zu diesem Zwecke bringt man neben Phosphor etwas Cadmiumsulfatlösung

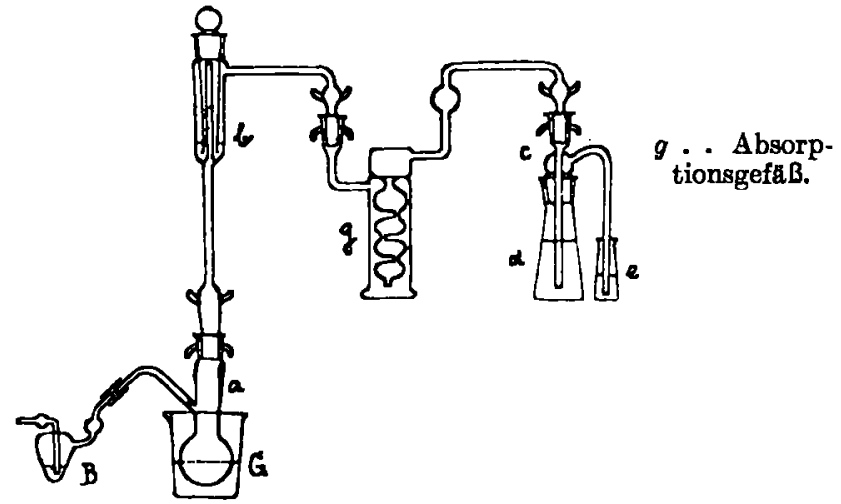

Abb. 2. (1/3 der natürlichen Größe.)

in den Wäscher. Bei Anwesenheit sehr großer Schwefelmengen genügt diese Anordnung nicht, da - selbst bei Verwendung von starker Cadmiumsulfatlösung - in der Silbernitratlösung der ersten und mitunter auch der zweiten Vorlage ein schwarzer Niedersohlag entsteht. In diesem Falle ist es notwendig, den Absorptionsapparat $g$ anzuwenden, der mit 30-40\% iger sehr sohwach angesäuerter Cadmiumsulfatlösung gefüllt wird ${ }^{6}$ ). Auf diese Weise wird der 4) Chem.-Ztg. 33, 264 [1909].

5) In den meisten Fällen kann man - bei Vermeidung eines allzu starken Kohlensäurestromes - von der Verwendung der zweiteu Vorlage absehen (und nur unter Benutzung des für Nachbestimmungen bestimmten Einleitungsröhrchens f arbeiten), namentlich wenn es sich um Glycerinbestimmungen in gleichartig zusammengesetzten Stoffen handelt, deren Verhalten im Jodidapparate bereits durch vorhergegangene Bestimmungen genügend erprobt ist.

-) Es ist empfehlenswert, auch bei Jodidbestimmungen von Stoffen minder großen Schwefelgehaltes den Absorptionsapparat zu verwenden, da der Wäscher durch das ausgeschiedene Cadmium- g e s a m t e Schwefel (im Gegensatz zu der Vorreinigung mit Bariumacetat, bei welcher nur der störende Einflu $B$ des $S$ u l f a $t$ schwefels beseitigt wird) von der Cadmiumsulfatlösung zurückgehalten, und man erhält ganz hellgefärbte Silberniederschläge.

Eine weitere Vereinfachung des Verfahrens besteht auch darin. da $B$ man, statt $0,5 \mathrm{ccm}$ einer verdünnten Glycerinlösung zu verwenden, die ursprüngliche Substanzdirekt in das Siedekölbchen einwägt und mit $1,5 \mathrm{ccm}$ Jodwasserstoffsäure (D. 1,9) versetzt. Um stark hygroskopische Stoffe auf diese Art behandeln zu können, wird das Siedekölbchen während der Wägung mit einem zum Schliffe passenden Stöpsel und das Seitenrohr des Kölbchens mit einer Kappe versehen. Das Eintragen der Probe gesohieht in diesem Falle mittels einer Tropfflasche mit eingeschliffener Pipette samt Kautschukhütchen. Für diese Art der Ausführung wird die Probe in einer Quantität zur Einwage herangezogen, die ungefähr 0,03-0,06 g Jodsilber liefert ${ }^{7}$ ). Stark wasserhaltige Proben, die gleichzeitig so glycerinarm sind, daß zur Analyse eine Einwage von mehr als 0,5 $\mathrm{g}$ erforderlich ist, werden vor der Behandlung im Jodidapparate im Siedekölbohen (bei höchstens $100^{\circ}$ ) so lange vorgetrocknet, bis der Wassergehalt bei Verwendung von etwa $4 \mathrm{~g}$ Einwage auf etwa $12 \%$, bei etwa $2 \mathrm{~g}$ Einwage auf etwa $25 \%$ und bei etwa $1 \mathrm{~g}$ Einwage auf etwa $50 \%$ gesunken ist.

Die Bestimmung selbst verläuft in genau derselben Weise wie im großen Jodidapparate, nur daB der Vorgang weit kürzere Zeit in Anspruch nimmt

Eine genaue Angabe der Ausführungsdauer ist nicht möglich, da die Zeit für die Behandlung im Jodidapparate von verschiedenen Umständen (wie Stärke des Siedens und des Kohlendioxydstromes und vor allem von der Natur der zu untersuchenden Substanz ${ }^{8}$ )) abhängig ist. Im allgemeinen jedoch ist eine Bestimmung, die einen normalen Verlauf nimmt, in etwa 50-60 Minuten beendet. Es ist aber jedenfalis anzuraten, sich durch Nachbestimmungen von der Beendigung der Operation zu überzeugen, besonders wenn das Verhalten der Probe im Jodidapparate noch unbekannter oder zweifelhafter Art ist.

Die weitere Behandlung des Silberniederschlages erfolgt in bekannter Weise, am besten nach der von $S$ tritar angegebenen Vorschrift. Wenn nach erfolgtem Waschen des Niederschlages im Filterröhrchen mit Wasser nooh ein Spülen mit Alkohol und hierauf mit Äther vorgenommen wird, ist für das Trocknen (im Trocken. schranke bei $120-130^{\circ}$ ) nur eine Zeit von 15-20 Minuten notwendig, so $\mathrm{da} \beta$ die Gesamtdauer für die vollständige Durchführung einer Glycerinbestimmung nach dem Halbmikrojodidverfahren ungefähr $21 / 2$ bis 3 Stunden beträgt.

3. Re a genzien. Für die Halbmikromethode werden dieselben Reagenzien (Phosphor, Jodwasserstoffsäure und Silbernitratlösung) von gleicher Beschaffenheit ${ }^{9}$ ) gebraucht und gelangen in derselben Konzentration wie bei Ausführung der Glycerinbestimmung im großen Apparate zur Anwendung. Es kommt nur noch die Cadmiumsulfatlösung hinzu, die $30-40 \%$ ig unter Zusatz weniger Tropfen verdünnter Sohwefelsäure hergestellt wird ${ }^{10}$ ),

4. Bedingungen und Behelfezur Ausführung der Halbmikrojodidbostimmung. a) Zum Ausführen von Bestimmungen nach dem Halbmikrojodidverf. ist vor allem eine gute, analytische Wage notwendig, die auf $0,1 \mathrm{mg}$ verläßlich empfindlich ist.

sulfid fortwährend beschmutzt wird und einer häufigen Reinigung bedarf, was jedoch jedesmal mit Verlusten an Phosphor verbunden ist.

7) Zur Orientierung über die für die Einwage erforderlichen Mengen der Probesubstanz mögen folgende Angaben dienen: Bei einem Glyceringehalt unter $1 \%$ sind etwa 2-4 g Einwage, bei einem Glyceringehalt von etwa $1 \%$ sind etwa 1 - $2 \mathrm{~g}$ Einwage, bei einem Glyceringehalt von etwa $10 \%$ sind etwa $0,2 \mathrm{~g}$ Einwage; bei einem Glyceringehalt von etwa $50 \%$ sind etwa $0,04 \mathrm{~g}$ Einwage, und kei konzentrierten Glycerinen von etwa $80-100 \%$ sind etwa $0,02 \mathrm{~g}$ Einwage notwendig.

$\left.{ }^{8}\right)$ So ist z. B. der Wassergehalt (oder die Konzentration) der Probesubstanz oder ein Gehalt an Zucker von Einfluß auf die Dauer der Jodidbestimmung. Auch scheint die Anwesenheit von Polyglycerinen, deren Umsetzung zu Isopropyljodid vermutlich nur langsam und allmählich erfolgt, verzögernd einzuwirken.

$\left.{ }^{8}\right)$ Bei Verwendung von Cadmiumsulfat ist allenfalls auch schwefelhaltige Jodwasserstoffsäure derselben Konzentration zulässig.

10) Bei Verwendung im Absorptionsapparate ist die Lösung in dieser Konzentration zu gebrauchen, zum Füllen des Wäschers kann sie derart verdünnt werden, daß eine etwa $10 \%$ ige Lösung entsteht. 
Der Gewichtssatz der Wage muß kontrolliert und bei merklichen Abweiohungen (mehr als 0,1- -0,2 mg) eine Korrektionstabelle angelegt werden.

Der Nullpunkt muß von Zeit zu Zeit nachgeprüft, und die erforderliohe Korrektur in Reohnung gezogen werden (was besonders dann zu geschehen hat, wenn die Wage auch für andere technische Analysen stark in Aaspruch genommen wird).

d) Die zar Verwendung gelangenden Meßgefäße müssen auf ihre Richtigkeit geprüft und erforderliohenfalls selbst geeicht werden. Dies geschieht am besten durch absolute Eichung mit Hilfo der Wage and unter Zugrundelegung desselben Gewichtssatzes, welcher für die Ausführung der Jodidbestimmungen benutzt wird. Auf diese Weise ist es möglich, Meßgefäße verschiedensten Rauminhaltes je nach Zweckmäligkeit beliebig zu gebrauchen. So wurden z. B. im hiesigen Laboratorium in den zahlreichen Fällen, in denen das Halbmikrojodidverfahren zur Anwendung gelangte, die zur Bestimmung erforderlichen 0,5 ccm einer Gesamtmenge von $10,15,25,50$ und $100 \mathrm{ccm}$ entnommen und stets einwandfrei tibereinstimmende Resultate erhalten.

Als einziges unbedingtes Erfordernis ist somit nur eine genau geeichte und sorgfaltig überprüfte $0,5 \mathrm{ccm}-\mathrm{P}$ i p e $t$ t e notwendig, die vollständig fettfrei sein und daher von Zeit zu Zeit mit saurer Kaliumbichromat- oder Permanganatlösung gewaschen werden muß11). Im hiesigen Laboratorium werden Pipetten mit birnförmiger Erweiterung von 0,5 com Inhalt und solche zylindrisoher Form von $1 \mathrm{com}$ oder $0,5{ }^{71} \mathrm{ccm}$ Inhalt (in 1/100 ccm geteilt) mit gleich gutem Erfolge verwendet.

c) Die Konzentration der glycerinhaltigen Lösungen, die zur Durchfïhrung der Jodidbestimmung in den Apparat gelangen, muß innerbalb jener Grenzen gewählt werden, die einerseits durch die nur auf $0,1 \mathrm{mg}$ empfindliohe Wage und andererseits duroh die für die Umsetzung zur Verfügung stehende Jodwasserstoffmenge gegeben sind. Es soll somit die Einwage der Probesubstanz derart bemessen werden, daß die Verdünnung auf ein bestimmtes Volumen eine durchschnittliche $2,5-5 \%$ ige Lösung ergibt, so da $B$ bei Entnahme von $0,5 \mathrm{ccm}$ zur Jodidbestimmung ungefähr $0,03-0,06 \mathrm{~g}$ Jodsilber zur Wägung gelangen.

Es wurden wohl auch glycerinärmere Lösungen (bis zu $0,5 \%$ ) dem Halbmikrojodidverfahren unterworfen und dabei ganz günstige Resultate erzielt; dooh ist im Interesse der Genauigkeit und Zuverlässigkeit des Verfahrens in der Regel von der Verwendung solch verdünnter Lösungen abzuraten.

Boi direkter Einwage der ursprünglichen Substanz in das Siedekölbchen muß die Menge der Probe ebenfalls derart gewählt werden, daß etwa 0,03-0,06 g Jodsilber entstehen.

d) Alle Operationen können bei Anwendung des Halbmikrojodidverfahrens in gewohnter Art und Weise vorgenommen werden. Besondere Sorgfalt ist nur auf das Messen der 0,5 ccm-Probelösung und auf dasWägen des Silberjodids zu verwenden.

5. Bereohnung des GI y cerins. Die Berechnung des Glycerins (in Prozenten ausgedrüokt) erfolgt nach der Formel:

$$
\text { Glyceringehalt }=\frac{a \times 0,3922}{\mathrm{E}} \times \mathrm{f} \times 100 \%
$$

wobei a das Gewicht des Jodsilbers, $f$ der, Verdünnungsfaktor und $\mathbf{E}$ die Einwage bedeutet.

6. B e l e g a n a l y s e n. Durch die vielen bisher ausgeführten Bestimmungen nach dem Halbmikrojodidverfahren konnte einwandfrei festgestellt werden, da B die Resultate, die manauf diese Art erbält, vollständig mit den Untersuohungsergebnissen nach dem Makrojodidverfahren übereinstimmen. Die kleinen Abweichungen liegen stets innerhalb der Fehlergrenzen, die auch bei Ausführung; der Jodidbestimmung im großen Apparate zulässig sind.

Als Beleganalysen mögen folgende Beispiele dienen:

1. Rine wässerige Glycerinlösung vom spez. Gew. 1,0212 (Lösung B).

11) Auch alle anderen für dieses Verfahren in Verwendung stehenden Meßgefäße, ebenso die Vorlagen, in denen sich der Silberniederschlag bildet, und die dazu gehörigen Einleitungsröhrchen sollen mög lichst fettfrei erhalten bleiben. Bei letzteren erleichtert dies sehr das Auswaschen des Niederschlages, der sich in diesem Falle nicht hartnäckig an den Wandungen festsetzt. (Im übrigen sei hier bemerkt, da $B$ am Einleitungsröhrchen anhaftende Beschläge leicht durch abwechselndes Spülen mit Wasser und Alkohol oder mit Hilfe eines kleinen Gummiwischers oder kleinen Bürstchens zu' entfernen sind.)
Gew öhnliche Jodid. bestim mung.

a) $20 \mathrm{ccm}$ der Lösung $B$ auf $100 \mathrm{com}$ verdünnt und $5 \mathrm{ccm}$ zur Jodidbestimmung verwendet.

Erhalten: 0,2291 g AgJ. Ergibt: 8,80\% Glycerin.

H a I b m ikrojodidbe b) $20 \mathrm{ccm}$ der Lösung $B$ auf $100 \mathrm{ccm}$ verdünnt und $0,5 \mathrm{ccm}$ zur Jodidbestimmung verwendet.

Erhalten: 0,0226 g AgJ (korr.). Ergibt: 8,68\% Glycerin.

c) $5 \mathrm{ccm}$ der Lösung $B$ auf $15 \mathrm{ccm}$ verdünnt und $0,5 \mathrm{ccm}$ zur Jodidbestimmung ver. wendet.

Erhalten: $0,0387 \mathrm{~g} \mathrm{AgJ}(0,0385 \mathrm{~g}$ korr.).

Ergibt: $8,92 \%$ Glycerin $(8,87 \%$ korr.).

2. Technisches, Glycerin enthaltendes Rohprodukt (sulfathaltig).

\section{A. Einwage: 8,0993 $\mathrm{g}$}

mit Wasser verdünnt, in der Hitze mit Bariumacetatlösung behandelt und nach dem Erkalton auf $100 \mathrm{ccm}$ aufgefült, geschüttelt und trocken filtriert.

a) 5 com des klaren Filtrats (b) 0,5 com des Filtrats zur Halbzur Jodidbestimmung vorwendet.

Erhalten: $0,4669 \mathrm{~g}$ AgJ.

Ergibt: 45,22\% Glyoerin.

mikrobestimmung verwendet.

Erhalten: $0,0466 \mathrm{~g} \mathrm{AgJ}$ (korr.).

Ergibt: 45,13\% Glycerin.

B. Einwage: $1,3375 \mathrm{~g}$,

in einem $15 \mathrm{ccm}-$ Kolben mit etwas Wasser verdiunnt, in der Würme mit Bariumacetatlösung bis zur vollständigen Ausfällung des Sulfats behandelt, naoh dem Erkalten auf $15 \mathrm{ccm}$ aufgefüllt, geschüttelt und durch ein trockenes Filter filtriert.

c) 0,5 com des klaren Filtrats zur Halbmikrobestimmung verwendet.

Erhalten: $0,0517 \mathrm{~g} \mathrm{AgJ}(0,0515 \mathrm{~g}$ korr.).

Ergibt: $45,48 \%$ Glycerin(45,30\% korr.).

7. $\mathrm{Z}$ u s a $\mathrm{m} m$ en $\mathrm{f}$ a s u $\mathrm{n}$ g. Im folgenden seien die wiohtigsten Anhaltspunkto zur Ausführung von Glycerinbestimmungen nach dem Halbmikrojodidverfahren kurz zusammengestellt.

I. Glyoerinbestimmug bei Abwesenhoit störender St offe

a) In Lösungen, die einen Glyceringehalt von ungefähr $2-5 \%$ besitzen

Man verwendet $0,5 \mathrm{ccm}$ der unveränderten lösung und unterwirft sie mit 1,5 ccm Jodwasserstoffsäure der Dichte 1,9 dem Jodidverfahren.

b) In Lösungen unter $2 \%$ Glyceringehalt:

Man bringt die Lösung durch Eindampfen auf die gewünsohte Konzentration (2,5-5\%), was zweckmäßig und rasch im Siedekölbohen selbst vorgenommen werden kann (indem man eine entsprechend größere Menge in das Kölbchen bringt, so daß durch Findampfen der Flüssigkeit auf ca, 0,5 ccm der gewünschte Konzentrationsgrad erreicht wird).

c) In Flüssigkeiten von mehr als $5 \%$ Glyceringehalt oder in festen glycerinhaltigen Körpern:

1. Durch Verdün nung einer entsprechenden Menge der Probe auf ein bestimmtes Volumen bzw. Lösen einer gewogenen Quantität des festen Stoffes in einem gemessenen Volumen Wasser, so daB eine Flüssigkeit von etwa 2,5-5\% Glyceringehalt entsteht, wovon $0,5 \mathrm{ccm}$ zur Jodidbestimmung heranzuziehen sind.

2. Durch direktes Ein wägen der flüssigen oder festen Probe ${ }^{12}$ ) (in das Siedekölbohen) in einer Quantität, die aus den Angaben auf Seite 235 ersichtlich ist, und deren Behandlung mit $1,5 \mathrm{ccm}$ Jodwasserstoffsäure (D . 1,9) im Jodidapparate. Utber die Verwendungsmöglichkeit von Jodwasserstoffsäure der Dichte 1,7 bei wasserärmeren, glycerinhaltigen Körpern sind noch Versuche im Gange.

In allen unter $\mathrm{I}$. angegebenen Fällen ist die Benutzung von Cadmiumsulfat nicht notwendig, jedoch keineswegs störend.

12) Diese Art wurde z. B. für Glycerinbestimmung in Seifen wiederholt mit Erfolg angewendet. 


\section{Glycorinbestim un ugbeiAnwesenheit schwefelhaltigerstoffe.}

a) Mit Vorreinigung.

I. Ohne Berücksichtigung des Bariumsulf a t volumens.

Ist der Schwefel nur in Form von Sulfat zugegen, kann in der bisher üblichen Weise vorgegangen und durch Behandeln der Probe mit Bariumacetat das Sulfat ausgefällt werden. Hierbei wird das Volumen des gebildeten Bariumsulfats vernachlässigt, was bei schwefelärmeren Substanzen keinen nennenswerten Fehler verursacht. Will man hingegen bei schwefelreicheren Körpern auch diesen Fehler vermeiden, so kann man - vorausgesetzt, daß man eine große Anzahl von Glyoerinbestimmungen in Stoffen annähernd gleichen Sulfatgehaltes auszuführen hat - die Analyse

2. mit Berücksichtigungdes Bariumsulfat v o l u m e n s durchführen, $d$. h. den Rauminhalt des Bariumsulfatniederschlages bei Verwendung einer bestimmten Einwage der Probe ein für allemal feststellen und bei der Berechnung des Glycerins in Abzug bringen ${ }^{13}$ ).

Im übrigen wird die vom Bariumsulfatniederschlage abgegossene oder trocken filtrierte) Lösung genau nach $P$ unkt I behandelt.

b) Ohne Vorreinigung.

Viel einfacher und rascher ausführbar ist die Durchführung der Jodidbestimmung in sohwefelhaltigen Substanzen bei Gegen wart von $\mathrm{C}$ a d $\mathrm{mi} \mathrm{u} \mathrm{m}$ s $\mathrm{l} f \mathrm{at}$ ohne vorhergehende Behandlung mit Bariumacetat, wobei auch kein Fehler durch Volumvernach. Jässigung begangen wird.

Auch in diesem Falle wird nach den im Punkte I ausgeführten Augaben verfahren, bloß mit dem Unterschiede, daß die Anwesenheit von Cadmiumsulfat - bei geringen Schwefelmengen im Wäscher, bei schwefelreicheren Stoffen im Absorptionsapparate - cine unbedingte Notwendigkeit ist.

Zur Ergänzung seien folgende Beleganalysen angeführt, die gleichzeitig die Richtigkeit dieses Arbeitsverfahrens erweisen sollen:

Verwendet wurde eine ungefähr 14\% Natriumsulfat enthaltende Substanz vom spez. Gew. 1,2337 (Probe S).

1. Zur Glycerinbestimmung wurden $20 \mathrm{ccm}$ der Probe $\mathrm{S}$ herangezogen, mit Bariumacetatlösung behufs Ausfällung des Sulfats behandelt und ohne Rücksicht auf den entstandenen Niederschlag a uf $100 \mathrm{ccm}$ gebracht. Die Jodidbestimmung wurde mit 0,5 ccm der klaren Lösung vorgenommen.

a) Erhalten: 0,0448 g AgJ (korr.).

Ergibt: 14,24\% Glycerin. . . . . . Resultat la (ohne Berücksichtigung des $\mathrm{BaSO}_{4}-$ - Vol.).

b) Das Resultat 1a mit Anbringung einer Korrektur, duroh die das Bariumsulfatvolumen in Rechnung gezogen wird, zeigt nachstehende Anderung:

Nach der Formel $x=R-G+a+b+K$ wurde ermittelt, daB bei Gegenwart von $3 \mathrm{~g}$ Natriumsulfat durch Behandlung mit Bariumacetat ein Bariumsulfatniedersohiag vom Volumen $2,8 \mathrm{ccm}$ entsteht, was bei Verwendung von 20 ccm der Substanz $S$ ungefähr zutrifft. Somit hat man bei Verdiunnung auf $100 \mathrm{com}$ nur ein Fiüssigkeitsvolumen von 97,2 ocm; daher ist bei Entnahme von $0,5 \mathrm{ccm}$ der Lösung zur Jodidbestimmung für die Berechnung des Glycerins statt des Verdünnungsfaktors 200 der korrigierte Wort 194,4 zu setzen (oder vom Glyceringehalte 14,24 sind $2,8 \%$ dieses Wertes in Abzug zu bringen), woraus sich ein wirklicher Glyceringehalt von $13,84 \%$ ergibt. . . . . . . . . . . . Resultat lb (mit Berücksichtigung des $\mathrm{BaSO}_{4}$-Vol. durch Anbringung einer Korrektur)

2. Zur Glycerinbestimmung "wurden wieder 20 ccm der Probe S verwendet, mit Bariumacetat behandelt, jedoch von Bariumsulfatniederschlage filtriert; der Niederschlag wurde am Filter sorgfaltig ausgewaschen und Filtrat samt Waschwasser bis auf etwa $80 \mathrm{ccm}$ eingeengt, hierauf in einem Moßkolben auf $100 \mathrm{ccm}$ gebracht. 0,5 ccm dieser Lösung wurden zur Jodidbestimmung herangezogen.

Erhalten: 0,0435 g Ag.J (korr.).

Ergibt: 13,83\% Glycerin. . . . . . . Resultat2\%(ohne Vernachlässigung des $\mathrm{BaSO}_{4}$-Vol.).

13) Das Volumen des Bariumsulfatniedersohlages bestimmt man, indem man in einen gewogenen Meßkolben vom Gewichte $K$ und
3. Glycerinbestimmung ohne Vorbehandlung mit Bariumacetat: $\mathrm{Zu}$ diesem $\mathrm{Zwe}$ we wurden wieder $20 \mathrm{com}$ der Probe $\mathrm{S}$ in einen $\mathrm{MeB}$ kolben gebracht und ohne Fällung mit Bariumacetat auf $100 \mathrm{ccm}$ verdünnt. Davon wurden $0,5 \mathrm{ccm}$ zur Jodidbestimmung entnommen, die bei Gegenwart von Cadmiumsulfat im Absorptionsapparate durchgeführt wurde.

Erhalten: 0,0434 g AgJ (korr.).

Ergibt: 13,80\% Glycerin. . . . . . Resultat 3 (ohne Vorbehandlung und ohne Volumvernachlässigung).

4. Glycerinhestimmung durch direkte Einwage der ursprünglichen Probe $\mathbf{S}$ in das Siedekölboben.

Einwage: $0,2097 \mathrm{~g}\left(0,17 \mathrm{ccm}^{3}\right)$, mit 1,5 ccm Jodwasserstoffsäure (D. 1,9) dem Jodidverfahren bei Gegenwart von Cadmiumsulfat im Absorptionsgefäße unterworfen.

Erhalten: $0,0738 \mathrm{~g} \mathrm{AgJ}$ (korr.).

Ergibt: 13,80\% Glycerin. . . . . . . Resultat 4/ohne Vorbehandlung durch direkte Einwage).

III. Glycerinbestimmung bei Anwesenheit von Chloriden.

Die Anwesenheit geringer Mengen von Chloriden ist ohne merklichen Einfluß a uf die mittels des Jodidverfahrens erhaltenen Glycerinwerte. Sind größere Mengen Chlor zugegen, so wird ein Mindergehalt von wenigen Zehntel Prozenten erhalten, ein Fehler, der bei technischen Analysen im allgemeinen vernachlässigt werden kann, umsomehr als die von $F$ a $n$ to angegebene Vorbehandlung ${ }^{14}$ ) behufs Vermeidung dieses Fehlers umständlich und sehr zeitraubend ist.

Somit erscheint es zweckmäßig, bei Anwesenheit von Chlor

1. die Bestimmung ohne Berücksichtigung des Chlors vorzu. nehmen und in derselben Weise, wie im Punkte I oder IIb ${ }^{15}$ ) angegeben ist, auszuführen, oder

2. das Chlorid mittels Silbersulfat oder Silberacetat zu fällen, das Volumen des gebildeten Silberchlorids zu vernachlässigen bzw. - bei Ausführung vieler Analysen in Stoffen annähernd gleichen Chlorgehaltes - eine Korrektur anzubringen, wodurch das Volumen des Silberchlorids (analog dem Bariumsulfatvolumen) in Abzug gebracht werden kann.

Im übrigen sind Versuche zwecks Vereinfachung der Behandlung chlorhaltiger Substanzen (direkte Bestimmung im Jodidapparate ohne Vorbehandlung unter gleichzeitiger Vermeidung von Fehlern durch Volumvernachlässigung) im Gange und derzeit nooh nicht abgeschlossen.

IV. Glycarinbestimmung bei Anwesenheit flüchtiger störender Stoffe.

Bei Anwesenheit von flüchtigen Alkoholen u. dgl. kann man die Vorbehandlung derart vereinfachen, daB man eine entsprechende Menge der Probe (0,5 ccm) in das Siedekölbchen bringt, mit $1 \mathrm{ccm}$ Wasser versetzt, durch Verdampfen der Flüssigkeit das ursprüngliche Volumen $(0,5 \mathrm{com})$ wieder herstellt und hierauf die Jodidbestimmung durchführt.

Das Halbmikrojodidverfahren verei nigt alle Vorzüge der Methode nach $Z$ e i sel-Fan to, ohne deren Nachteile - die eingangs erwbhnt wurden - zu besitzen. Die Kosten einer Bestimmung sind a uf ungefähr den zehnten Teil und die Ausführungszeit (ohne die Vorreinigung nach Z e i e 1 - F a n to in Rechnung zu ziehen) auf etwa die Hälfte vermindert.

Rauminhalte $\mathrm{R}$ eine gewogene Menge ohemisch reines Bariumchlorid (a Gramm) bringt und die zur Fällung notwendige Menge Natriumsulfat (b Gramm) hinzufügt, bis zur Marke mit destilliertem Wasser auffüllt und das Gesamtgewicht $G$ feststellt. Das Volumen des aus a Grammen Bariumohlorid entstandenen Bariumsulfatniederschlages $\mathbf{x}$ bereohnet man nach folgender Gleichung: $\mathbf{x}=\mathrm{R}-\mathrm{Q}+\mathbf{a}+\mathbf{b}$ $+\mathrm{K}$ com

14) Fällen mit Silbersulfat, hierauf mit Barumacetat, Filtrieren und quantitatives Auswaschen des Niedersohlages, Einengen des mit dem Waschwasser vereinten Filtrates usw. (Angew. Chem. 16, 413 [1903].)

15) Nach Punkt I, wenn kein Sohwefel anwesend ist, nach IIb, wenn neben Chlor auch Sohwefel vorhanden ist. 
Aus nachfolgender Zusammenstellung ist der Preisunterschied der Materialien deutlich zu entnehmen:

\begin{tabular}{|c|c|c|}
\hline & Jodidverfahren & Halbmlkrojodidverfahren \\
\hline $\begin{array}{c}\text { Preise } \\
\text { der Materialien in } \\
\text { Friedenszeiten }\end{array}$ & $\begin{array}{l}15 \mathrm{ccm} \mathrm{HJ}(\mathrm{D} .1 .9) 1,80 \mathrm{~K} . \\
2 \mathrm{~g} \mathrm{AgNO}, \cdot 0,18, \\
\frac{45 \mathrm{ccm} \text { Alkohol } \cdot 0,16 "}{2,14 \mathrm{~K}}\end{array}$ & 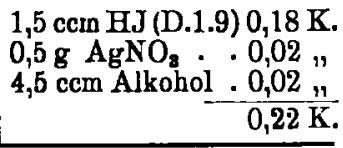 \\
\hline Jetzige Preise & $\left|\begin{array}{l}15 \mathrm{ccm} \text { HJ(D. 1.9) } 2,70 \mathrm{~K} . \\
2 \mathrm{~g} \text { AgNO } \\
45 \mathrm{ccm} \text { Alkohol } \cdot 0,60,35, \\
\frac{3,65 \mathrm{~K}}{}\end{array}\right|$ & 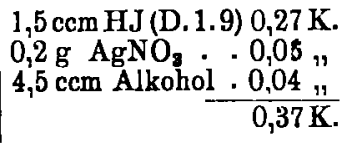 \\
\hline
\end{tabular}

Diese Kosten beziehen sich überdies nur auf die Chemikalien, ohne dabei die Zeit und die Arbeitskraft zu berücksichtigen.

Eine weitere Ausnutzung der Zeit besteht auch darin, daß man eine Anzahl von Bestimmungen gleichzeitig nebeneinander - bei Verwendung mehrerer Apparate, die in Form einer Batterie geschaltet sein können, so daß die Kohlensäure einer einzigen Stahlflasche entnommen wird - und ohne Unterbrechung hintereinander - bei Auswechslung von Siedekölbchen und Vorstoß samt Vorlagen an je einem Apparate - ausführen kann. Bei dieser Arbeitsweise eignet sich das Jodidverf. auch für den Massenbetrieb, indem bei gleichzeitiger Verwendung von 3 Jodidapparaten, täglich - bei achtstündiger Arbeitszeit - ungefähr 9 Glycerinbestimmungen vollständig durchgeführt werden können.

[A. 74.]

\section{Verfahren zur kostenlosen direkten Gewinnung chemisch reiner Salpetersäure in Verbindung mit der Valentiner-Methode zur Darstellung roher Salpetersäure.}

Von Dr. Johanwes Thedr, Engis (Belgien).

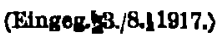

Ti. Unter chemisch reiner Salpetersëure wird eine Säure verstanden, deren Trockenrückstand nicht mehr ajs $0,0015 \%$ betrïgt, und die frei von Chlor-, Jod-, Untersalpeter- und Schwefelsäure ist. Eine so beschaffene Siure entsprioht auch den von der Pharmakopöe gestellten Bedingungen.

Im allgemeinen wurde die Rektifikation der rohen Salpetersäure bisher durch nochmalige Destillation in Glas- oder Bleigefäßen bewirkt. Nach dem D. R.P. $155006 \mathrm{zm}$ B. fließt die zu reinigendo Salpetersäure auf Kieselsteine oder dgl., die in cinem von außen geheizten Destillierkessel eingefüllt sind. Die Säure soll verdampfen, bevor sie die Gefäßwandungen berührt, und dann in beliebiger Weise k-ndensiert werden. Die nicht flüchtigen Verunreinigungen, wie Eisenverbindungen, Kieselsäure, Bisulfat, sollen in dem Kessel zurückbleiben. Die bekannte Methode von $P$ ützer arbeitet mit Bleigefäßen, die sich in einem durch ein Kohlenfeuer beheizten Chlorcalciumbad befinden. Die bei 120 bis $130^{\circ}$ überdestillierende Säure wird in Liebigkühlern konden siert. Durch Einblasen von Luft muB das Destillat von der reichlich auftretenden Untersalpetersäure befreit werden. Trotzdem dieses Verfahren als eine Verbesserung der früher üblichen Reinigungsmethoden angesehen wird, ist es noch recht umständlich und erfordert erhebliche Unkosten. Diese belaufen sich auf rund 10,00 M für $100 \mathrm{~kg}$ fertiger Säure $36 \mathrm{er}$, wie an einem Beispiel gezeigt werden soll. Die Jahresproduktion der chemischen Fabrik Friedri o h Curti u s in Duisburg a. $R h$. an chemisch reiner Salpetersäure von $36^{\circ}$ Bé betrug im Jahre $191097049 \mathrm{~kg}$. Für ihre Herstellung waren folgende Ausgaben erforderlich:

I. Konzentrationskosten für die Überführung der Aus-

gungssäure $=102871 \mathrm{~kg} 36 \mathrm{er}$ in $48 \mathrm{er}$ Săure $.=3303,44 \mathrm{M}$

2. Säureverlust: $5822 \mathrm{~kg} 36$ er Săure 18,5 . . . . = = 1077,07,

3. Kohlen: $43710 \mathrm{~kg} \times 1,2 \ldots . . . . . . .=524,52$ "

4. Fabrikationslöhne . . . . . . . . . . . . . . . . $=1548,96$,

5. Reparaturlöhne . . . . . . . . . . . . . . . . = 367,47,

6. Reparaturmaterialien, wio Asbest, Schrauben,

Flanschen, Muttern, Gummiteile, Glaswaren, Ton-

waren usw. ............. . . = 1226,23,

7. Salzsäure, Kalk . . . . . . . . . . . . . . . . = 202,35,

Zus.: 8250,04 M
Übertrag: $8250,04 \mathrm{M}$

8. Destilliertes Wasser, etwa $40000 \mathrm{~kg}$. . . . . . . = 96,00 , 9. Aufsicht, Luftpumpen, Verschiedenes . . . . . . = 500,00",

10. Zinsen, und Amortisation des Anlagekapitals von

$4000 \mathrm{M} \mathrm{.} \mathrm{.} \mathrm{.} \mathrm{.} \mathrm{.} \mathrm{.} \mathrm{.} \mathrm{.} \mathrm{.} \mathrm{.} \mathrm{.} \mathrm{.} \mathrm{.} \mathrm{.}=800,00$,

also für $100 \mathrm{~kg}$ chemisch reiner Säure $9,94 \mathrm{M}$.

Zus. : $=9646,04 \mathrm{M}$

Dagegen wird nach dem Verfahren, das von dem Verfasser dieses Aufsatzes unter der wertvollen Beihilfe des verstorbenen Fabrikbesitzers R i c hard Curti u s, und des Fabrikmeisters $\mathrm{J}$ a k o b Fox, beide in Duisburg a. Rh., ausgearbeitet wurde, die chemisch reine Säure völlig kostenlos gewonnen. Die Methode, die vornehmlich auf Anwendung der fraktionierten Destillation beruht, scheidet aus der nach dem Valentinerverfahren erhaltenen Rohsäure einen gewissen Teil direkt als chemisch reine Säure aus. Die Ausbeute kann bis zu $20 \%$ der Gesamtsäure betragen. Das Verfahren beruht auf der Beobachtung, da $B$ die ersten und letzten Fraktionen der Rohsäure das meiste Chlor enthalten, das sind im allgemeinen die über $44^{\circ}$ Bé. und die unter $40^{\circ}$ Bé. Der genaue Punkt, wo die überdestillierende Säure chlorfrei ist, und der Augenblick, wo sie wieder anfüngt, chlorhaltig zu werden, müssen bei jeder Destillation durch fortlaufende qualitative Analysen festgelegt werden. Die chlorfreie Säure, also im allgemeinen die zwischen $44-40^{\circ} \mathrm{Bé}$, wird gesondert aufgefangen; sie erweist sich als chemisch rein. Sollte es vorkommen, daß sich in der genannten Fraktion Spuren von Chlor finden, so hat man nur nötig, diese etwa eine Stunde lang mit filtrierter Luft zu blasen. Die Temperatur der Säure beträgt dabei am besten gegen $50^{\circ}$. An einem besonderen Beispiel soll gezeigt werden, wie dieses Verfahren, und zwar mit bestem Erfolg, in der Praxis zur Ausübung gekommen ist. Es ist hierbei noch eine Reihe von Bedin. gungen einzuhalten, um in jedem Fall das gewünschte Resultat zu erreichen. Es hat sich gezeigt, daB, je größer die angewandte Destillationsblase ist, um so sicherer chemisch reine Säure erhalten wird. Der Unterteil der Retorte hatte einen Inhalt von $18 \mathrm{cbm}$ und war zur Aufnahme einer Charge von $8000 \mathrm{~kg}$ Salpeter geeignet. Zur Verwendung kamen raffinierter Salpeter, der bis $0,6 \% \mathrm{NaCl}$ enthielt, und 60 er rohe Gloversäure mit einem Gehalt bis zu 0,4\% As, außer anderen Verunreinigungen. Vor allem ist es wichtig, die Destillation so gleichmäßig wie möglich zu leiten. Aus diesem Grunde erfolgte die Beheizung der Retorte mittels einer Muffelfeuerung, so daß eine direkte Berührung zwischen dem Retortenboden und der Flamme nicht stattfinden konnte. Die Temperatur der überdestillierenden Säure wurde möglichst niedrig gehalten; sie sollte $160^{\circ}$, gemessen beim Austritt aus der Retorte, nicht übersteigen. Als bestes Vakuum wurde das zwischen $300-400 \mathrm{~mm}$ Quecksilbersäule ermittelt. Die zur Verwendung gelangenden Kondensationsgefäße und Kühlschlangen bestanden aus gebranntem Steinzeug, die teils von den Deutschen Ton- und Steinzeugwerken in Charlottenburg, teils von den Sohamottewerken in Euskirchen geliefert waren. Ein Unterschied bezüglich ihres Widerstandes gegen Salpetersüure wurde nicht festgestellt. Bevor sie für diese Gewinnung chemisch reiner Salpetersäure benutzt wurden, dienten sie für die Kühlung und Kondensation von roher Salpetersäure, die aus mindestens 80-100 Operationen zu $8000 \mathrm{~kg}$ Salpeter resultierte. Was also etwa von diesen Schlangen und GefäBen löslich war, hatte die Salpetersüure herausgelöst. Man hatte es nun mit Behältern zu tun, die in technischem Sinne als völlig unempfindlich gegen Salpetersäure zu betrachten waren. Ob die Verwendung derartig ausgelaugter Gefäße für das Gelingen des Verfahrens eine Notwendigkeit ist, vermag nicht gesagt zu werden. Jedenfalls wurde festgestellt, daß neue Tontöpfe verhältnismäßig stark von Salpetersäure angegriffen werden.

Wie oben ausgeführt, betragen die Kosten für die Überführung der rohen Salpetersäure in chemisch reine Säure nach dem Verfahren von $P \ddot{u} t z$ er rund $10,00 \mathrm{M}$. Andere bisher übliche Methoden werden nicht billiger arbeiten. Der materielle Nutzen, den die geschilderte Neuerung abwirft, liegt darum klar auf der Hand und dürfte vor allem diejenigen Firmen zu einem Versuohe veranlassen, die eine Salpetersäureanlage nach dem Valentine rschen System besitzen. Damit soll nicht gesagt sein, da $\beta$ nicht auch andere Fabriken, die nach $G$ uttma $n$, Ư bel usw. die Salpetersäure gewinnen, unter zweckmäßiger Abänderung des Verfahrens Erfolg mit der direkten Herstellung chemisch reiner Salpetersäure haben sollten. Hicrzu möchte der vorstehende Aufsatz die Anregung gegeben haben. Ob allerdings der Luftsalpeter für die Zukunft nicht andere Wege für die Gewinnung chemisch reiner Salpetersäure weisen wird, mag dahingostellt bleiben. 